\title{
Immune activation
}

\section{suppresses plasma testosterone level:} a meta-analysis

\author{
Jelle J. Boonekamp ${ }^{1}$, Albert H. F. Ros ${ }^{2}$ \\ and Simon Verhulst ${ }^{1, *}$ \\ ${ }^{1}$ Behavioural Biology, University of Groningen, PO Box 14, \\ 9750 AA Haren, The Netherlands \\ ${ }^{2}$ Eco-Ethology Research Unit, ISPA, Lisbon 1149-041, Portugal \\ *Author for correspondence (s.verhulst@rug.nl).
}

Females often select mates on the basis of sexual signals, which can be reliable indicators of male quality when the costliness of these signals prevents cheating. The immunocompetence handicap hypothesis (ICHH) provides a mechanistic explanation of these costs, by proposing a trade-off between immune function and sexual displays. This trade-off arises because testosterone enhances sexual signals, but suppresses immune function. Many studies have investigated the ICHH by administrating testosterone, and a recent meta-analysis found little evidence that testosterone suppressed immune function. However, another component of the ICHH, which has received less empirical interest, suggests that there may also be an interaction in the other direction, with immune activation suppressing testosterone levels. We present a meta-analysis to test for this effect. Overall, there was a strong suppressive effect of experimental immune activation on testosterone levels $(r=-0.52)$, regardless of whether live pathogens or non-pathogenic antigens were used to challenge the immune system. The latter is important because it shows that immune activation per se suppresses testosterone levels. Thus, a trade-off between immunocompetence and sexual displays may primarily be generated by the effect of immune activation on testosterone, rather than the opposite effect that has received most attention.

Keywords:

immunocompetence handicap hypothesis;

sexual selection; testosterone; sexual signals

\section{INTRODUCTION}

Males that have more intense sexual signals often have higher mating success (Andersson 1994), which has raised the question of what constrains sexual signals. Hamilton \& Zuk (1982) hypothesized that the expression of sexual signals reflects health and vigour, but did not specify what mechanism could constrain the development of sexual signals by individuals in poor health. Folstad \& Karter (1992) filled this gap by proposing the immunocompetence

Electronic supplementary material is available at http://dx.doi.org/ 10.1098/rsbl.2008.0347 or via http://journals.royalsociety.org. handicap hypothesis (ICHH), which summarized what was then known about the interactions between sexual signals, androgens, parasites and the immune system. Their main prediction was that there would be a trade-off between sexual displays on one hand and immune function on the other hand. This prediction was primarily based on the supposed dual effect of testosterone ( $\mathrm{T})$, which enhances sexual displays, but suppresses immune function (Folstad \& Karter 1992). Thus, only males that can afford to suppress their immune system, for example, because they are genetically well adapted to the prevailing parasites, are able to maintain sexual signals at high levels.

The ICHH has played an important role in shaping the study of parasite-mediated sexual selection, and many studies have been carried out to test this hypothesis or its assumptions. The main focus of the ICHH and the studies it inspired has been on the supposed dual effect of T. Studies that tested the assumption that $\mathrm{T}$ suppresses immunocompetence were meta-analysed by Roberts et al. (2004), who found that this effect was on average small and far from statistically significant. On the other hand, a meta-analysis of the effect of parasites on sexual signals, another component of the $\mathrm{ICHH}$, revealed that experimental exposure to parasites significantly suppressed sexual signals (Møller et al. 1999). Since the expression of sexual signals is often regulated by testosterone, this suggests that parasites or the immune system may suppress T levels (Hillgarth \& Wingfield 1997; Verhulst et al. 1999). This was part of the ICHH as originally formulated, but has received comparatively little attention. In this paper, we present a meta-analysis that investigates the effect of immune activation on $\mathrm{T}$. In view of the result of Roberts et al. (2004) that on average $\mathrm{T}$ does not suppress immunocompetence, it would be important to confirm whether immune activation suppresses $T$, since this would be an alternative mechanism that generates a trade-off between immunocompetence and sexual signals.

\section{MATERIAL AND METHODS}

We searched experimental studies that included an in vivo immune challenge followed by plasma $\mathrm{T}$ measurements in adult male subjects using electronic databases and by checking references of relevant papers. In total, 13 eligible studies were found (600 individuals of six different species; table 1). It is worth noting that these 13 publications were scattered throughout the literature, and only two studies were couched in a sexual selection framework. One publication contained two independent experiments and we treated these as independent data points.

Effect sizes were expressed as correlation coefficients $(r)$. Unless mentioned otherwise, we used studies as units of analysis, but also report results using species as units of analysis. A random model was used to test and quantify effect sizes using 'Comprehensive Meta-Analyses' (v. 2).

For further details of the literature search and effect size calculations see the electronic supplementary material.

\section{RESULTS}

Immune challenges suppressed $\mathrm{T}(r=-0.52 ; 95 \% \mathrm{CI}$ : $-0.61,-0.41 ; p<0.001)$, also when using species as units of analysis $(r=-0.45$; 95\% CI: $-0.53,-0.37$; $p<0.001)$. Publication bias can distort meta-analyses but a funnel graph suggests little bias (figure 1), and a trim-and-fill test did not change the overall effect size. Furthermore, Rosenthal's fail-safe $N$-test (Rosenthal 1984) revealed that 520 studies with a mean effect size 
Table 1. Effect sizes of immune challenge on plasma $\mathrm{T}$ levels. (Immune challenges with live pathogens are marked with an asterisk.)

\begin{tabular}{|c|c|c|c|c|c|}
\hline species & immune challenge & sample size $(n)$ & effect size $(r)$ & $p$-value & reference \\
\hline \multicolumn{6}{|l|}{ mammals } \\
\hline mouse & lps & 55 & -0.442 & 0.0007 & 1. Hales et al. (2000) \\
\hline mouse & malaria* & 16 & -0.711 & 0.002 & 2. Barthelemy et al. (2004) \\
\hline mouse & schistosoma* & 42 & -0.827 & $<0.0001$ & 3. Isseroff et al. (1986) \\
\hline mouse & schistosoma* & 18 & -0.708 & $<0.001$ & 4. He et al. (2000) \\
\hline mouse & $\operatorname{lps}$ & 62 & -0.575 & $<0.0001$ & 5. Weil et al. (2006) \\
\hline rabbit & schistosoma* & 16 & -0.261 & 0.335 & 6. Kasilima et al. (2004) \\
\hline rat & lps & 92 & -0.513 & $<0.0001$ & 7. O'Bryan et al. (2000) \\
\hline rat & lps & 136 & -0.386 & $<0.0001$ & 8. O'Bryan et al. (2000) \\
\hline sheep & malaria* & 9 & -0.516 & 0.155 & 9. Mutayoba et al. (1997) \\
\hline \multicolumn{6}{|l|}{ birds } \\
\hline collared flycatcher & SRBC & 13 & -0.584 & 0.036 & 10. Garamszegi et al. (2004) \\
\hline chicken & bronchitis* & 16 & -0.497 & $<0.05$ & 11. Boltz et al. (2004) \\
\hline chicken & bronchitis* & 19 & -0.511 & 0.169 & 12. Boltz et al. (2007) \\
\hline chicken & mite ${ }^{*}$ & 26 & -0.388 & $<0.05$ & 13. De Vaney et al. (1977) \\
\hline chicken & SRBC & 80 & -0.328 & 0.003 & 14. Verhulst et al. (1999) \\
\hline overall effect & & 600 & -0.517 & $<0.0001$ & \\
\hline
\end{tabular}

of 0 are required to render the observed effect nonsignificant. Thus, we conclude that the outcome of our analysis is unlikely to be due to publication bias.

There was significant heterogeneity among effect sizes $(Q=27.9, p=0.009)$, which could imply that in subgroups immune activation did not suppress T. We therefore quantified the overall effect size separately, grouping the data with respect to two prominent factors. Immune activation was achieved using a living or a non-living immune challenge, and both yielded significant effect sizes (figure $2 a$ ). Likewise, the effect sizes of birds and mammals were both significant (figure $2 b$ ). In neither case did the subgroups differ from each other $(p>0.35)$. We repeated the analyses in figure 2 using species as units of analysis, which for technical reasons necessitated restriction to one of the four subgroups in figure 2 per analysis. This did not change the conclusions $(-0.58 \leq r \leq-0.36$, all $p<0.0001)$.

\section{DISCUSSION}

Overall, there was a strong T-suppressive effect of immune activation (figure 1). This conclusion appears robust, in the sense that the fail-safe test and the funnel plot suggest that it is unlikely to be caused by publication bias. Furthermore, it holds independent of taxonomy, antigen type and data treatment, at least within the limited range used in the available studies (figure 2). Physiological pathways mediating the immune effect on $\mathrm{T}$ may vary depending on the nature of the immune challenge, e.g. modulate gonadal activity via the hypothalamus (Ogilvie \& Rivier 1998; Klein 2004), or affect the testis directly, either via the nervus vagus or via blood-borne messengers such as cytokines (Bornstein et al. 2004). Unfortunately, the available data do not yet allow further investigation of the importance of such variation for effect sizes.

Parasitized individuals have less elaborate sexual signals than healthy individuals (Møller et al. 1999), which may be mediated by a T-suppressive effect of parasite infection. This effect could arise indirectly, via

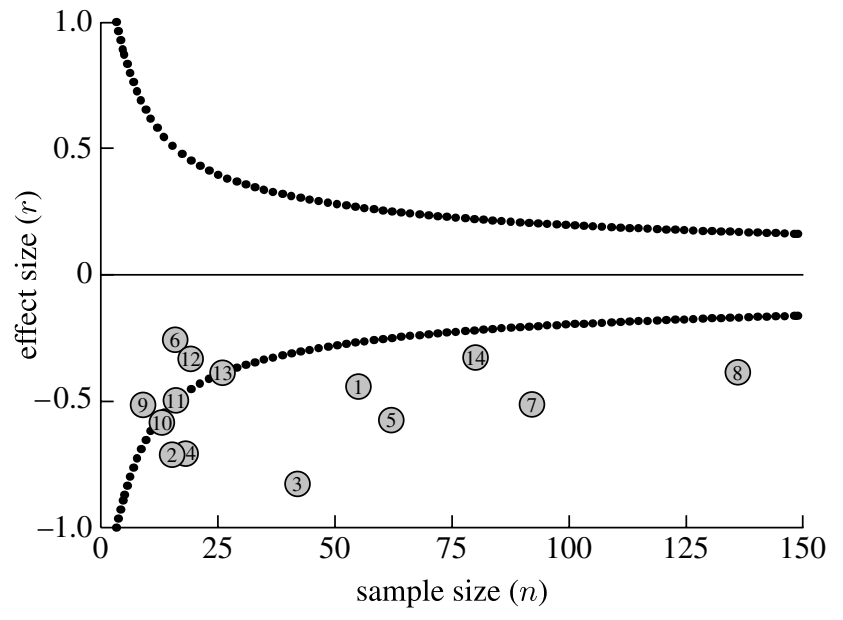

Figure 1. Funnel plot of effect size against sample size. Dotted lines show values at which $r$ differs significantly from zero with $p=0.05$. Numbers correspond to studies in table 1 .

a parasite effect on host health and vigour when this results in lower $\mathrm{T}$, or, not mutually exclusive, could be a direct effect of immune activation, channelling resources from signalling to health maintenance using $\mathrm{T}$ as regulating hormone. It is important in this respect that our analysis showed that an immune challenge using non-living antigens also suppressed $\mathrm{T}$ (figure $2 a$ ), indicating that $\mathrm{T}$ suppression is part of the host response to an infection. This conclusion is in agreement with in vivo experiments showing that cytokine IL-1 suppresses steroid production by Leydig cells (e.g. Hales 1992; Svechnikov et al. 2001; Lister \& van der Kraak 2002).

Much attention has been paid to the assumption of the ICHH that $\mathrm{T}$ has a dual effect on immunocompetence and sexual signals, but perhaps a more interesting aspect of the ICHH is the prediction that this mechanism leads to a trade-off between immunocompetence and sexual signalling. The finding of Roberts et al. (2004) that there is on average no immunosuppressive effect of $\mathrm{T}$ (although there may 

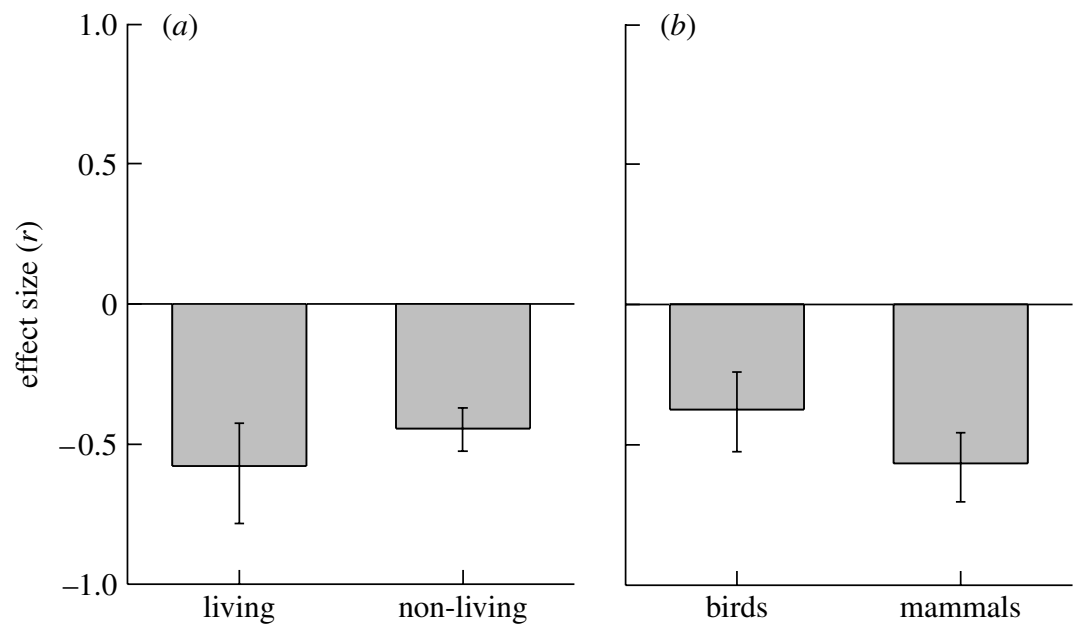

Figure 2. Mean effect size $( \pm 95 \% \mathrm{CI}$ ) of immune challenges on $\mathrm{T}$ for subgroups (all $p<0.001)$. (a) Immune challenge type: living (parasites, virus) versus non-living. (b) Taxonomic group: birds versus mammals.

well be such an effect in specific situations) provides a challenge for this prediction, since without such an effect the level of $\mathrm{T}$ (and thus sexual signalling) can be increased without impeding the immune system. However, the opposite pathway we examine in this paper, of immune activation suppressing $\mathrm{T}$, also produces a trade-off between immunocompetence and sexual signalling. Thus, we agree with the ICHH prediction that there is a trade-off between immunocompetence and sexual signalling, but suggest that this trade-off is primarily generated by the effect of immune activation on testosterone, rather than the opposite effect that has received most attention. Parasite prevalence is often substantial (e.g. Hellgren et al. 2008), and given the plethora of parasite species there are probably very few individuals completely free from infection. Thus, many individuals may permanently experience the T-suppressive effect of immune activation, and females can use T-dependent displays to select a healthy mate.

Hongbin He provided unpublished statistical details of their study. Ivar Folstad, Michael Jennions and Ton Groothuis provided comments that improved the manuscript. S.V. was supported by a NWO-VICI grant, and A.H.F.R. by the Portuguese Foundation for Science and Technology.

Andersson, M. 1994 Sexual selection. Princeton, NJ: Princeton University Press.

Barthelemy, M., Gabrion, C. \& Petit, G. 2004 Reduction in testosterone concentration and its effect on the reproductive output of chronic malaria-infected male mice. Parasitol Res. 93, 475-481. (doi:10.1007/s00436-0041160-2)

Boltz, D. A., Nakai, M. \& Bahr, J. M. 2004 Avian infectious bronchitis virus: a possible cause of reduced fertility in the rooster. Avian Dis. 48, 909-915. (doi:10.1637/7192040808R1)

Boltz, C. R., Boltz, D. A., Bunick, D., Scherba, G. \& Bahr, J. M. 2007 Vaccination against the avian infectious bronchitis virus affects sperm concentration, sperm quality and blood testosterone concentrations in cockerels. B. Poult. Sci. 48, 617-624. (doi:10.1080/000716 60701592375)
Bornstein, S. R., Rutkowski, H. \& Vrezas, I. 2004 Cytokines and steroidogenesis. Mol. Cell Endocrin. 215, 135-141. (doi:10.1016/j.mce.2003.11.022)

De Vaney, J. A., Elissalde, M. H., Steel, E. G., Hogan, B. F. \& Delvarpetersen, H. 1977 Effect of northern fowl mite, Ornithonyssus sylviarum on white leghorn roosters. Poult. Sci. 56, 1585-1590.

Folstad, I. \& Karter, A. J. 1992 Parasites, bright males, and the immunocompetence handicap. Am. Nat. 139, 603-622. (doi:10.1086/285346)

Garamszegi, L. Z., Møller, A. P., Torok, J., Michl, G., Peczely, P. \& Richard, M. 2004 Immune challenge mediates vocal communication in a passerine bird: an experiment. Behav. Ecol. 15, 148-157. (doi:10.1093/ beheco/arg108)

Hales, D. B. 1992 Interleukin-1 inhibits Leydig-cell steroidogenesis primarily by decreasing 17-alpha-hydroxylase C17-20 lyase cytochrome P450 expression. Endocrinology 131, 2165-2172. (doi:10.1210/en.131.5.2165)

Hales, K. H., Diemer, T., Ginde, S., Shankar, B. K., Roberts, M., Bosmann, H. B. \& Hales, D. B. 2000 Diametric effects of bacterial endotoxin lipopolysaccharide on adrenal and Leydig cell steroidogenic acute regulatory protein. Endocrinology 141, 4000-4012. (doi:10.1210/en. 141.11.4000)

Hamilton, W. D. \& Zuk, M. 1982 Heritable true fitness and bright birds: a role for parasites? Science 218, 384-387. (doi:10.1126/science.7123238)

He, H. B., Ohta, N. \& Kawaguchi, H. 2000 Effect of schistosoma japonicum infection on serum testosterone levels in mice. Zhongguo fi Sheng Chong Xue Yu fi Chong Bing Za Zhi. 18, 173-175.

Hellgren, O., Bensch, S. \& Malmqvist, B. 2008 Bird hosts, blood parasites and their vectors-associations uncovered by molecular analyses of blackfly blood meals. Mol. Ecol. 17, 1605-1613. (doi:10.1111/j.1365-294X.2007. 03680.x)

Hillgarth, N. \& Wingfield, J. C. 1997 Parasite-mediated sexual selection: endocrine aspects. In Host-parasite evolution: general principles and avian models (eds D. H. Clayton \& J. Moore), pp. 78-104. Oxford, UK: Oxford University Press.

Isseroff, H., Sylvester, P. W. \& Held, W. A. 1986 Effects of Schistosoma mansoni on androgen regulated geneexpression in the mouse. Mol. Biochem. Parasitol. 18, 401-412. (doi:10.1016/0166-6851(86)90096-4)

Kasilima, Y. S., Wango, E. O., Kigondu, C. S., Mutayoba, B. M. \& Nyindo, A. 2004 Plasma bioactive LH and 
testosterone profiles in male New Zealand rabbits experimentally infected with schistosoma mansoni. Acta Trop. 92, 165-172. (doi:10.1016/j.actatropica.2004.06.004)

Klein, S. L. 2004 Hormonal and immunological mechanisms mediating sex differences in parasite infection. Parasite Immunol. 26, 247-264. (doi:10.1111/j.0141-9838.2004. 00710.x)

Lister, A. \& van der Kraak, G. 2002 Modulation of goldfish testicular testosterone production in vitro by tumor necrosis factor alpha, interleukin-1 beta, and macrophage conditioned media. F. Exp. Zool. 292, 477-486. (doi:10.1002/jez.10066)

Møller, A. P., Christe, P. \& Lux, E. 1999 Parasitism, host immune function, and sexual selection. Q. Rev. Biol. 74, 3-20. (doi:10.1086/392949)

Mutayoba, B. M., O'Shaughnessy, P. J., Jeffcoate, I. A., Eckersall, P. D., Cestnik, V. \& Holmes, P. H. 1997 Effect of experimental infection with Trypanosoma congolense and scrotal insulation on Leydig cell steroidogenesis in the ram. Theriogenology 48, 411-422. (doi:10.1016/ S0093-691X(97)00251-3)

O'Bryan, M. K., Schlatt, S., Phillips, D. J., de Kretser, D. M. \& Hedger, M. P. 2000 Bacterial lipopolysaccharide-induced inflammation compromises testicular function at multiple levels in vivo. Endocrinology 141, 238-246. (doi:10.1210/en.141.1.238)
Ogilvie, K. \& Rivier, C. 1998 The intracerebroventricular injection of interleukin-1 beta blunts the testosterone response to human chorionic gonadotropin: role of prostaglandin- and adrenergic-dependent pathways. Endocrinology 139, 3088-3095. (doi:10.1210/en.139.7. 3088)

Roberts, M. L., Buchanan, K. L. \& Evans, M. R. 2004 Testing the immunocompetence handicap hypothesis: a review of the evidence. Anim. Behav. 68, 227-239. (doi:10.1016/j.anbehav.2004.05.001)

Rosenthal, R. 1984 Meta-analytic procedures for social research. Beverly Hills, CA: Sage Publication.

Svechnikov, K. V., Sultana, T. \& Soder, O. 2001 Agedependent stimulation of Leydig cell steroidogenesis by interleukin-1 isoforms. Mol. Cell. Endocrin. 182, 193-201. (doi:10.1016/S0303-7207(01)00554-8)

Verhulst, S., Parmentier, H. K. \& Dieleman, S. J. 1999 A trade-off between immunocompetence and sexual ornamentation in domestic fowl. Proc. Natl Acad. Sci. USA 96, 4478-4481. (doi:10.1073/pnas. 96.8.4478)

Weil, Z. M., Bowers, S. L., Pyter, L. M. \& Nelson, R. J. 2006 Social interactions alter proinflammatory cytokine gene expression and behavior following endotoxin administration. Brain Behav. Immun. 20, 72-79. (doi:10. 1016/j.bbi.2005.05.001) 